\title{
Orçamentação nos hotéis do Algarve: Alinhamento com a prática internacional
}

\section{Insights and international alignment of budgeting practices in the Algarve region}

\author{
Ana Rita Faria \\ Escola Superior de Gestão, Hotelaria e Turismo, Universidade do Algarve, Portugal \\ arfaria@ualg.pt \\ Leonor Ferreira \\ Nova School of Business and Economics, Portugal \\ leonor.ferreira@novasbe.pt \\ Duarte Trigueiros \\ ISTAR-IUL, University Institute of Lisbon, Portugal \\ duarte.trigueiros@iscte-iul.pt
}

\begin{abstract}
Resumo
Os orçamentos são um instrumento essencial para planeamento e controlo, avaliação do desempenho, comunicação, coordenação e motivação do pessoal. A sua utilização no setor hoteleiro é generalizada; contudo, ainda pouco conhecida em Portugal. Este estudo analisa as práticas de orçamentação dos hotéis do Algarve. Dados recolhidos por meio de questionários a responsáveis financeiros revelam uma elevada utilização dos orçamentos. Estes são elaborados essencialmente para um horizonte anual e servem principalmente para avaliar o desempenho, definir objetivos e metas, auxiliar o controlo e planear a curto prazo. Poucos hotéis elaboram orçamentos flexíveis e orçamentos de base zero. Os orçamentos são um dos principais indicadores de desempenho. Os resultados obtidos estão em linha com a literatura de contabilidade hoteleira e com a prática internacional.
\end{abstract}

Palavras-chave: Orçamentos; práticas de orçamentação; processo orçamental; contabilidade de gestão; setor hoteleiro; Algarve.

\begin{abstract}
Budgets are an essential tool for planning and control, performance evaluation, communication, coordination and staff motivation. Despite their importance and wide use in the hotel sector, research into the budgeting practices of hotels located in Portugal is scarce. This study analyses the budgeting practices of hotels in the Algarve. Data collected through a structured questionnaire submitted to financial managers shows that budgets are widely adopted; however, they are mainly produced for the short term. The primary reasons for preparing budgets are performance evaluation, target setting, control and short-term planning. Few hotels use zero-base budgeting and there is also little use of flexible budgeting. Budgets are viewed as one of the main performance indicators. The findings are in line with the conclusions of previous studies.
\end{abstract}

Keywords: Budgets; budgeting practices; budgeting process; management accounting; hotel sector; Algarve.

Dos Algarves: A Multidisciplinary e-Journal, $34-2019$.

ISBN 2182-5580 @ ESGHT - University of the Algarve, Portugal.

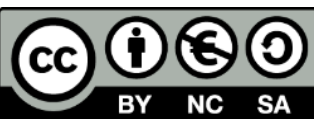

To cite this article: Faria, A. R. Ferreira, L. \& Trigueiros, D. (2019). Orçamentação nos hotéis do Algarve: Alinhamento com a prática internacional. Dos Algarves: A Multidisciplinary e-Journal, 34, 3-25. doi: 10.18089/DAMeJ.2019.34.1 


\section{Introdução}

As empresas operam atualmente num ambiente incerto e em constante mutação, o que lhes exige que, para minimizarem o risco dos investimentos e tomarem decisões atempadas, efetuem permanentemente previsões. Os orçamentos são uma ferramenta fulcral no processo de planeamento e controlo das organizações (Uyar \& Bilgin, 2011; Joshua \& Mohammed, 2013), sendo igualmente reconhecido o seu papel como instrumento de comunicação, coordenação, motivação do pessoal, controlo e avaliação do desempenho. Admite-se que os orçamentos sejam a técnica de contabilidade de gestão mais antiga e a mais utilizada (Dugdale, 1994; Chenhall \& Langfield-Smith, 1998; Joshi, 2001; Ferreira, 2002; Jones, 2006; Gomes, 2007; Santos, Gomes \& Arroteia, 2010). No entanto, os orçamentos têm sofrido severas críticas, sendo-lhes apontadas diversas fraquezas (Bunce, Fraser \& Woodcock, 1995; Hope \& Fraser, 2003). No início do presente século, Hope e Fraser (2003) propuseram mesmo o seu abandono. Em contraponto, estudos recentes revelam que o processo orçamental continua a acrescentar valor às organizações (Libby \& Lindsay, 2010).

Nas duas últimas décadas, vários autores alertaram para a necessidade de investigação sobre as práticas de orçamentação no setor dos serviços, em particular no setor hoteleiro (Schmidgall, Borchgrevink \& Zahl-Begnun, 1996; Jones, 1998; Sharma, 2002; Cruz, 2007; Uyar \& Bilgin, 2011; Souza \& Lunkes, 2015). Embora o recente interesse pela contabilidade de gestão hoteleira se tenha traduzido num volume considerável de publicações sobre os sistemas de contabilidade de gestão nos hotéis, a investigação nesta área é limitada e tem incidido nos países emissores de turismo, tais como os Estados Unidos da América (EUA), a Escandinávia e o Reino Unido, mas menos nos países recetores, como é o caso da Grécia (Makrigiannakis \& Soteriades, 2007) e Portugal. Denota-se também uma escassez de investigação nos países em desenvolvimento (Uyar \& Bilgin, 2011; Souza \& Lunkes, 2015). Na Turquia, na Eslovénia e no Brasil vieram recentemente a lume estudos sobre práticas de orçamentação nos hotéis, mas a aplicação dos orçamentos no setor hoteleiro português está ainda por explorar. Este estudo amplia assim o conhecimento na área, ao caracterizar exaustivamente as práticas de orçamentação dos hotéis de quatro e cinco estrelas de um dos principais destinos turísticos portugueses: o Algarve. Assim, este trabalho tem como objetivo verificar se estas práticas estão alinhadas com a prática internacional, designadamente no tocante à elaboração de orçamentos, ao seu horizonte temporal, às razões para a elaboração dos orçamentos, à antecedência com que se inicia o processo de elaboração do orçamento anual, à abordagem utilizada, ao tipo de informação utilizada na elaboração do orçamento anual, à utilização de orçamentos de base zero e de orçamentos flexíveis, à revisão dos orçamentos e à sua relevância na avaliação do desempenho.

O estudo dá primazia aos aspetos técnicos do processo orçamental, não ignorando, porém, a importância da vertente comportamental na consecução dos objetivos organizacionais e no êxito da componente técnica do processo. Na indústria hoteleira, os aspetos humanos dos sistemas de orçamentação podem considerar-se especialmente relevantes em face da sua orientação para "as pessoas", reconhecendo-se que os orçamentos e outros controlos contabilísticos não devem ser simplesmente processos técnicos (Harris \& Brown, 1998). Entre os estudos que analisam a vertente humana dos sistemas de orçamentação e de controlo contam-se os de Brown (1995), Jones (2006) e Uyar e Bilgin (2011). 
O estudo encontra-se estruturado da seguinte forma: na Secção 2 revê-se a literatura sobre práticas de contabilidade de gestão e orçamentação nos hotéis. Na Secção 3 enunciamse as questões de investigação e a metodologia de investigação utilizada. Na Secção 4 discutem-se os resultados. Finaliza-se, na Secção 5, com uma breve conclusão e sugestões para investigação futura.

\section{Revisão da literatura}

\subsection{Da utilização de orçamentos}

Os orçamentos - expressão quantitativa de planos de ação global propostos pelos gestores e elaborados para um dado período - são a técnica de contabilidade de gestão mais utilizada (Jones, 2006). Comprovam-no diversos estudos conduzidos em vários países e em diversos setores de atividade.

Ainda na década de noventa do século passado, na Grã-Bretanha, Dugdale (1994) verificou que os profissionais atribuíam grande relevância à orçamentação, especialmente para o planeamento, embora conferissem importância reduzida a técnicas sofisticadas como a orçamentação de base zero. Também Chenhall e Langfield-Smith (1998), na Austrália, destacaram a importância das técnicas de planeamento tradicionais, designadamente a orçamentação e o planeamento a longo prazo.

Já no presente século, na India, Joshi (2001) confirmou que as empresas confiam profundamente em técnicas tradicionais como a orçamentação e a avaliação do desempenho por meio de indicadores financeiros.

Também em Portugal, Ferreira (2002) e Gomes (2007) observaram que os orçamentos e a análise de desvios orçamentais são as técnicas tradicionais mais utilizadas nas empresas do setor não financeiro (indústria e serviços) e em grandes empresas de setores diversos, respetivamente.

Em investigação mais recente realizada na América do Norte (médias e grandes empresas), Libby e Lindsay (2010) notam que os orçamentos continuam a ser amplamente utilizados para fins de controlo e a maioria das organizações tenciona continuar a utilizá-los com esta finalidade. Embora lhe reconheçam problemas, as organizações planeiam alterar e adaptar o sistema de orçamentação ao invés de o abandonarem.

Estudos aplicados ao setor hoteleiro em diversos países mostram que, nos hotéis, os orçamentos são também amplamente utilizados e reconhecidos como uma importante ferramenta de controlo de gestão.

Na Grécia, segundo Makrigiannakis e Soteriades (2007) e Pavlatos e Paggios (2009), as práticas de orçamentação são extensamente adotadas nos hotéis.

Em Portugal, Santos et al. (2010) observaram que a orçamentação e a análise dos desvios orçamentais são as técnicas tradicionais de contabilidade de gestão mais utilizadas pelos hotéis. A contabilidade de gestão serve essencialmente para apoiar a tomada de decisão e o processo orçamental.

Joshua e Mohammed (2013), na Nigéria, confirmaram que a maioria dos hotéis utiliza orçamentos no planeamento das atividades.

Em estudo mais recente realizado no Brasil, Souza e Lunkes (2015) também constataram que a maioria dos hotéis adota o orçamento empresarial. 


\subsection{Práticas de orçamentação no setor hoteleiro}

No Quadro 1 apresentam-se estudos sobre orçamentação no setor hoteleiro.

Quadro 1. Estudos empíricos sobre orçamentação no setor hoteleiro

\begin{tabular}{lll}
\hline Autor(es) (data) & \multicolumn{1}{c}{ País/Região } & Amostra \\
\hline Schmidgall \& Ninemeier (1987) & Estados Unidos & Cadeias hoteleiras \\
\hline Schmidgall et al. (1996) & $\begin{array}{l}\text { Estados Unidos e } \\
\text { Escandinávia }\end{array}$ & Hotéis e cadeias hoteleiras \\
\hline Jones (1998, 2006) & Reino Unido & Organizações hoteleiras \\
\hline Schmidgall \& DeFranco (1998) & Estados Unidos & Responsáveis financeiros \\
\hline Uyar \& Bilgin (2011) & Turquia (Antalya) & $\begin{array}{l}\text { Hotéis de 3, 4 e 5 estrelas e apart- } \\
\text { hotéis }\end{array}$ \\
\hline Souza \& Lunkes (2015) & $\begin{array}{l}\text { Brasil (10 regiões } \\
\text { turísticas) }\end{array}$ & $\begin{array}{l}\text { Hotéis de várias categorias e } \\
\text { tipologias }\end{array}$ \\
\hline
\end{tabular}

Os estudos que versam especificamente as práticas de orçamentação hoteleiras escasseiam; os que existem utilizam como método de investigação o questionário. Com elevada frequência, a temática surge integrada em trabalhos de âmbito mais vasto, quando se examina a utilização das várias técnicas de contabilidade de gestão (e.g., Collier \& Gregory, 1995; Planas, 2004; Cruz, 2007; Makrigiannakis \& Soteriades, 2007; Pavlatos \& Paggios, 2009; Santos et al., 2010) ou outros temas (e.g., Ivankovic \& Jerman, 2011; Joshua \& Mohammed, 2013). A maioria dos estudos tem origem nos EUA (Schmidgall \& Ninemeier, 1987; Schmidgall et al., 1996; Schmidgall \& DeFranco, 1998) ou no Reino Unido (Collier \& Gregory, 1995; Jones, 1998, 2006). Mais recentemente surgiram estudos noutras geografias, nomeadamente na Turquia (Uyar \& Bilgin, 2011), na Eslovénia (Ivankovic \& Jerman, 2011), na Nigéria (Joshua \& Mohammed, 2013) e no Brasil (Souza \& Lunkes, 2015).

Os trabalhos identificados cobrem usualmente aspetos como: horizonte dos orçamentos, razões para a elaboração de orçamentos, antecedência com que se inicia o orçamento, participação no processo orçamental, informação utilizada na elaboração dos orçamentos, orçamentação de base zero, orçamentos flexíveis, revisão e controlo orçamental. Seguidamente procede-se à análise dos mesmos.

\subsubsection{Planeamento e preparação dos orçamentos}

2.2.1.1. Horizonte dos orçamentos: $\mathrm{Na}$ indústria hoteleira, o processo orçamental envolve duas atividades interligadas: o orçamento de longo prazo e o orçamento anual (Collier \& Gregory, 1995; Schmidgall et al., 1996; Schmidgall \& DeFranco, 1998; Cruz, 2007).

No Reino Unido, Collier e Gregory (1995) constataram que o orçamento de longo prazo abrange um período de três a cinco anos. O orçamento anual divide-se em doze períodos mensais ou em treze períodos de quatro semanas e segue os procedimentos dos livros de texto, incluindo orçamentos detalhados para os principais departamentos (Alojamento, Alimentação e Bebidas, Comunicações e Outros custos) e para as principais áreas de custos 
indiretos (Serviços Gerais e Administrativos, Marketing, Energias e Manutenção e Reparação). o orçamento de longo prazo inclui o Balanço, a Demonstração dos Resultados e a Demonstração dos Fluxos de Caixa e é atualizado anualmente numa base contínua; um verdadeiro expediente de planeamento estratégico, que permite à gestão analisar o impacto de uma variedade de estratégias sob diferentes cenários.

Igualmente no Reino Unido, Jones $(1998,2006)$ verificou que mais de $90 \%$ dos hoteleiros elaboram orçamentos para um período financeiro; a restante minoria elabora orçamentos para um período de seis meses ou para um período contínuo de doze meses.

Já em Portugal, na joint venture analisada por Cruz (2007), constituída para operar e gerir 30 hotéis sob a marca de uma cadeia internacional, os gestores hoteleiros preparam orçamentos operacionais para o ano seguinte, no total e por departamentos (e.g., Alojamento, Alimentação e Bebidas). O planeamento a longo prazo é feito ao nível da sede e traduz-se em planos de negócios para cinco anos, que contemplam atividades não rotineiras, como sejam novos investimentos.

$\mathrm{Na}$ Grécia, os orçamentos também abrangem geralmente um ano. Como notam Makrigiannakis e Soteriades (2007: 71), “[... ] a proporção de hotéis gregos que utiliza orçamentos de longo prazo é mais baixa na Grécia do que em qualquer outra parte”.

Na Turquia, Uyar e Bilgin (2011) observaram igualmente que três quartos dos hotéis elaboram orçamentos anuais essencialmente para um período de doze meses.

Nos EUA, segundo Schmidgall e DeFranco (1998), o orçamento operacional é parte integrante da operação financeira dos hotéis, mas menos de metade orçamenta para além de um ano. A idêntica conclusão chegaram Schmidgall e Ninemeier (1987), na década anterior, ao verificarem que apenas $53,3 \%$ dos hóteis estabeleciam previsões a mais de um ano (período mais utilizado: cinco anos).

Schmidgall et al. (1996) por seu turno, notaram uma tendência para o planeamento a longo prazo nos EUA ( $76 \%$ dos casos preparavam orçamentos para cinco ou mais anos), sem correspondência nos hotéis da Escandinávia já que aí somente 26,9\% preparavam orçamentos operacionais para este período e um terço para três anos.

Na Eslovénia, segundo Ivankovic e Jerman (2011), a maioria dos hotéis possui uma estratégia de negócio a longo prazo e, por conseguinte, um orçamento. Os hotéis mais bemsucedidos definem a estratégia para três anos. Concluem ainda que a existência de estratégias a longo prazo está relacionada com a eficácia dos hotéis.

No Brasil, $42 \%$ dos hotéis adotam o orçamento empresarial e $58 \%$ elaboram-no para o período de um ano (Souza \& Lunkes, 2015).

2.2.1.2. Razões para elaborar orçamentos: Os hotéis elaboram orçamentos essencialmente para auxiliar o controlo e avaliar o desempenho, como constataram Jones $(1998,2006)$ no Reino Unido e Makrigiannakis e Soteriades (2007) na Grécia. Neste país, muitos hotéis também utilizam os orçamentos para comunicar e estabelecer metas (Makrigiannakis \& Soteriades, 2007), planear as operações anuais e coordenar as atividades dos diversos segmentos organizacionais (Pavlatos \& Paggios, 2009).

Nos EUA, a maioria dos hotéis usa os orçamentos principalmente como padrão (standard) para comparação com o desempenho real e como ferramenta de planeamento. As cadeias nacionais preparam o orçamento para análises comparativas, mais 
frequentemente do que os independentes; estes preferem usar o orçamento operacional como uma ferramenta de gestão (Schmidgall \& DeFranco, 1998).

$\mathrm{Na}$ Turquia, os hotéis preparam orçamentos essencialmente para controlar custos, analisar a rendibilidade e auxiliar o planeamento a longo prazo. Motivar os empregados e comunicar planos são as razões menos invocadas (Uyar \& Bilgin, 2011).

No Brasil, os principais motivos da orçamentação são maximizar os resultados, planear, monitorizar e avaliar os resultados e apoiar o controlo orçamental a curto prazo (Souza \& Lunkes, 2015).

2.2.1.3. Antecedência na elaboração do orçamento anual: Há três décadas, nos EUA, Schmidgall e Ninemeier (1987) verificaram que o orçamento se iniciava com cinco meses de antecedência. Cerca de 10 anos depois, Schmidgall et al. (1996) observaram que mais de três quartos dos hotéis dos EUA e cerca de $70 \%$ dos hotéis da Escandinávia iniciavam o processo orçamental com uma antecedência mais curta: entre três e cinco meses. Schmidgall e Ninemeier (1987) constataram ainda que o número de níveis do grupo tem um nítido efeito sobre a duração do calendário orçamental: quanto maior o seu número, maior a duração.

No Reino Unido, Jones (2006) verificou que o processo orçamental se inicia com uma antecedência de três meses. Contrariamente ao estudo de Schmidgall e Ninemeier (1987), este estudo sugere que a dimensão (mais unidades e mais níveis) não é um fator determinante pois existe a consciência de que a tecnologia moderna permite carregar dados de dez ou cem hotéis em simultâneo.

Em Espanha, Planas (2004) observou que as cadeias internacionais iniciam o processo com maior antecedência (terceiro trimestre do exercício anterior). As cadeias espanholas mais pequenas seguem um processo de elaboração mais tardio, sendo que $12 \%$ chegam a elaborar os orçamentos no mês de dezembro e $25 \%$ já depois de iniciado o próprio exercício.

Na Turquia, Uyar e Bilgin (2011) verificaram que a maioria dos hotéis leva em média 1,78 meses a preparar o orçamento anual.

Já no Brasil, Souza e Lunkes (2015) observaram que o tempo gasto na preparação do orçamento é reduzido, envolvendo 40 horas em $40 \%$ dos hotéis e somente mais de 120 horas em apenas $12 \%$.

2.2.1.4. Participação no processo orçamental: Em teoria, o processo orçamental pode seguir a abordagem top-down, bottom-up ou uma combinação de ambas.

No Reino Unido, em 1998, Jones constatou que a abordagem bottom-up era utilizada por quase $55 \%$ dos inquiridos. Mas, posteriormente, Jones (2006) observou um decréscimo de aproximadamente $20 \%$ no número de organizações que reportaram utilizá-la. Para a autora, apesar de a indústria acreditar que a participação é essencial no processo orçamental, a abordagem bottom-up não é eficiente pois consome demasiado tempo e pressupõe a confiança nos gestores dos níveis inferiores da organização, os quais nem sempre possuem formação capaz para gerar um orçamento eficaz. Assim, segundo Jones (2006), a abordagem "combinação" é aquela que permite manter o controlo da direção estratégica ao estabelecer primeiro objetivos e metas globais e só depois permitir que os gestores, ao nível da unidade, contribuam com o input para uma orçamentação mais pormenorizada a nível departamental. 
Ainda no Reino Unido, Collier e Gregory (1995) não encontraram evidências de abordagens top-down ou bottom-up puras. A maioria dos casos segue um processo iterativo, sendo o orçamento iniciado no hotel e posteriormente negociado com a sede.

$\mathrm{Na}$ Grécia, regra geral, os hotéis utilizam a abordagem top-down, exercendo os gestores intermédios alguma influência (Makrigiannakis \& Soteriades, 2007).

Na Turquia, $42 \%$ dos hotéis utiliza uma combinação de abordagens e 34\% a abordagem bottom-up; apenas 24\% utiliza a abordagem top-down (Uyar \& Bilgin, 2011).

No Brasil, Souza e Lunkes (2015) verificaram ser a abordagem bottom-up a mais utilizada (40\% dos hotéis); as abordagens top-down e "combinação" são utilizadas em 25\% e 18\% dos hotéis, respetivamente.

Na Escandinávia, segundo Schmidgall et al. (1996), a abordagem predominante nas cadeias hoteleiras é a bottom-up (64\%); contudo, apenas um terço dos hotéis individuais utiliza esta abordagem. Os autores concluem que "nos hotéis escandinavos, a gestão de topo parece «ditar» mais do que parece estar a acontecer nos EUA" (Schmidgall et al: 193) e admitem que a dimensão dos hotéis escandinavos possa ser a principal razão para a preferência por esta abordagem.

Em Portugal, a organização analisada por Cruz (2007) segue a abordagem bottom-up. $\mathrm{O}$ processo tem início nos hotéis e nunca na sede pois admite-se que os diretores gerais têm um maior conhecimento das operações no terreno. Os orçamentos operacionais são depois agrupados a níveis superiores até ao nível do país, e subsequentemente negociados com a empresa-mãe.

Em suma, nos hotéis nota-se uma preferência pela abordagem bottom-up ou por uma combinação das duas abordagens tradicionais.

2.2.1.5. Informação utilizada na elaboração dos orçamentos: No Reino Unido, Jones (2006) constatou que praticamente todos os hotéis se apoiam nos valores reais do ano anterior, sendo estes secundados por análises de mercado feitas internamente, indicadores económicos locais e estatísticas e indicadores da indústria. Os valores orçamentados do ano anterior são pouco utilizados por não acrescentarem valor ao processo, especialmente num ambiente em rápida mudança.

$\mathrm{Na}$ Turquia, Uyar e Bilgin (2011) observaram igualmente que os indicadores mais amplamente utilizados são os resultados reais dos anos anteriores, as estatísticas/indicadores da indústria e análises de mercado feitas internamente. Também na Eslovénia, os hotéis utilizam mais frequentemente os custos de períodos anteriores (52,8\%) como base para os orçamentos do período seguinte; logo depois, os custos estimados. Os custos padrões são pouco utilizados (Ivankovic \& Jerman; 2011). Souza e Lunkes (2015) verificaram que, também no Brasil, os orçamentos são elaborados com base essencialmente nos resultados reais de anos anteriores e em indicadores económicos.

Relativamente aos valores usados nas previsões de vendas, aspeto aflorado por Schmidgall et al. (1996), mais de 90\% dos hotéis norte-americanos e escandinavos recorrem igualmente a informação histórica. Já os hotéis gregos ponderam fatores como o impacto de alterações nos preços, informação operacional histórica, indicadores económicos nacionais e dispêndios em marketing. A maioria dos hotéis considera ainda indicadores económicos locais, enquanto metade analisa também indicadores económicos internacionais (Makrigiannakis \& Soteriades, 2007). 
2.2.1.6. Orçamentação de base zero: A orçamentação de base zero, que exige que todos os valores sejam construídos a "partir do zero" e justificados, não é a regra na indústria hoteleira.

Metade das organizações do Reino Unido não recorre à orçamentação de base zero (Jones, 1998, 2006). Trata-se de uma ferramenta a utilizar somente em novos hotéis, onde não existem dados históricos. A justificação é que não compensa o tempo adicional envolvido. Aqueles que utilizam a orçamentação de base zero consideram-na mais valiosa para orçamentar custos do que receitas (Jones, 2006).

Segundo Schmidgall et al. (1996), a técnica é utilizada por $42 \%$ dos hotéis americanos e apenas por $7 \%$ dos hotéis escandinavos. A principal aplicação pelos primeiros é nos departamentos auxiliares. Em Espanha, os orçamentos de base zero não são utilizados pelas cadeias hoteleiras e apenas $16,7 \%$ dos outros hotéis os elabora (Planas, 2004). Também na Turquia esta técnica é pouco utilizada; somente $12 \mathrm{em} 79$ hotéis a adotam (Uyar \& Bilgin, 2011).

Na Nigéria, apenas 30\% dos hotéis utilizam a técnica (Joshua \& Mohammed, 2013); no Brasil, a proporção de hotéis que a ela recorrem é diminuta: 3\% (Souza \& Lunkes, 2015).

Porém, na Grécia, Makrigiannakis e Soteriades (2007) e Pavlatos e Paggios (2009) reportam taxas de utilização relativamente elevadas, $60 \%$ e $49,4 \%$, respetivamente. Segundo Makrigiannakis e Soteriades (2007), os hotéis elaboram orçamentos de base zero, essencialmente para calcular os custos dos departamentos operacionais, o que contraria os resultados de Schmidgall et al. (1996).

Em Portugal, Cruz (2007) observou que a orçamentação de base zero cedeu lugar, em 2004, à orçamentação incremental. Um procedimento segundo o qual, "os objetivos orçamentais anuais se baseiam nos valores do ano anterior e em que se redigem planos de ação para justificar as diferenças em face do desempenho do ano anterior (após se efetuarem ajustamentos para eventos extraordinários)" (Cruz, 2007: 582).

2.2.1.7. Orçamentos flexíveis: No Reino Unido, Collier e Gregory (1995) verificaram que nenhuma organização hoteleira elabora orçamentos flexíveis, não calculando assim desvios de quantidade. O reduzido custo marginal em todas as linhas de negócio, a elevada proporção dos custos fixos na indústria e a ideia de que as receitas são a prioridade foram as razões apontadas. O resultado é consistente com Fitgerald et al. 1990 (apud Collier \& Gregory, 1995), que observaram que, embora fletir o orçamento para o atual número de clientes fosse desejável, a elevada base de custos fixos mitiga as vantagens de ajustar o orçamento.

Ainda no Reino Unido, Jones $(1998,2006)$ constatou que apenas $16 \%$ e 22,6\%, respetivamente, das organizações inquiridas utilizam a técnica. Contudo, as que não a utilizam regulamente, reconhecem-Ihe utilidade em circunstâncias excecionais, como o 11 de Setembro (Jones, 2006). Corroborando Collier e Gregory (1995), a autora sublinha que provavelmente haverá pouco a ganhar com a orçamentação flexível na indústria hoteleira dada a elevada base de custos fixos e acrescenta que a técnica terá maior utilidade em situações de custos variáveis elevados. Este fator, conjugado com o elevado custo de produzir um orçamento flexível, leva a crer que esta ferramenta não seja eficaz no tocante aos custos. 
No Brasil, a técnica é também pouco utilizada; em apenas 13\% dos hotéis (Souza \& Lunkes, 2015).

Contrariamente, na Grécia, segundo Makrigiannakis e Soteriades (2007), a elaboração de orçamentos flexíveis é comum, encontrando-se os custos ligados ao volume de vendas. A sazonalidade justifica que assim seja, pois força os hotéis a elaborarem os orçamentos muitos meses antes do retomar das operações. Para os autores, um orçamento standard com um distanciamento de quatro meses das primeiras chegadas não pode ser considerado uma ferramenta fiável. Contudo, um estudo posterior de Pavlatos e Paggios (2009) reporta que apenas $15,3 \%$ dos hotéis gregos elabora orçamentos flexíveis.

Na Turquia, Uyar e Bilgin (2011) observaram igualmente taxas elevadas; quase $70 \%$ dos hotéis utilizam a orçamentação flexível.

\subsubsection{Revisão e controlo do orçamento}

2.2.2.1. Revisão do orçamento: Os orçamentos são elaborados com alguma antecedência e num contexto operacional dinâmico vários fatores podem ter impacto no orçamento e "forçar" a sua revisão (Schmidgall et al., 1996). Um ataque terrorista, uma doença contagiosa ou alterações climáticas podem influenciar a indústria hoteleira muito rapidamente (Uyar \& Bilgin, 2011).

Neste aspeto, os resultados de Schmidgall e Ninemeier (1987) e de Schmidgall et al. (1996) para os EUA não divergem: 70\% dos hotéis americanos reveem os orçamentos. $\mathrm{Na}$ Escandinávia, segundo Schmidgall et al. (1996) apenas 44\% dos hotéis o fazem. O processo começa nos primeiros três meses do período orçamentado em quase $62 \%$ dos hotéis norteamericanos e num terço dos escandinavos. Nos EUA prevalece a revisão mensal e na Escandinávia a revisão trimestral. No estudo de 1987, Schmidgall e Ninemeier também haviam notado que a maioria dos reforecasts era feita numa base mensal ou trimestral sendo depois o orçamento revisto para o resto do ano fiscal. Porém, Schmidgall e DeFranco (1998) observaram que apenas $25 \%$ revia o orçamento durante o ano, embora a frequência de alteração mais comum fosse a mensal (40\%).

Na Europa, a maioria dos hotéis gregos, segundo Makrigiannakis e Soteriades (2007) revê os orçamentos durante o ano, essencialmente com a finalidade de efetuar previsões e avaliar o desempenho. Jones (1996), por seu turno, verificou que metade das organizações revê o orçamento através de reforecasts, mas mesmo nessas circunstâncias, o orçamento original continua a ser utilizado na avaliação do desempenho. Uyar e Bilgin (2011), por seu turno, constataram que quase metade dos hotéis revê o orçamento trimestralmente e 30\% fazem-no semestralmente. Na Eslovénia, a maioria dos hotéis adapta mensalmente os custos à escala das operações; cerca de $23 \%$ fazem-no trimestralmente (Ivankovic \& Jerman, 2011). Em Portugal, na organização estudada por Cruz (2007), a meio do ano é preparado um orçamento contínuo (rolling budget), que revê o orçamento operacional anual para os seis meses seguintes. Mas, apesar do orçamento anual ser revisto duas vezes por ano, à semelhança do que relata Jones (2006), o bónus dos diretores continua a basear-se na comparação dos valores reais com os valores orçamentados inicialmente.

No Brasil, $25 \%$ dos hotéis revê o orçamento trimestralmente, $22 \%$ mensalmente e $18 \%$ a cada seis meses; metade dos hotéis não faz alterações resultantes de variações no volume (Souza \& Lunkes, 2015). 
2.2.2.2. Controlo orçamental: Em geral, reconhece-se a valia dos orçamentos como ferramenta de controlo nos hotéis (Schmidgall \& Ninemeier, 1987; Brown, 1995; Collier \& Gregory, 1995; Schmidgall \& DeFranco, 1998; Jones, 1998, 2006; Schmidgall et al., 1996; Cruz, 2007; Makrigiannakis \& Soteriades, 2007). Schmidgall e Ninemeier (1987), nos EUA, e Schmidgall et al. (1996), nos EUA e na Escandinávia, verificaram que a tendência é limitar a responsabilidade dos gestores apenas aos custos sob o seu controlo.

Quanto ao nível aceitável de desvios entre o orçamento e o desempenho atual, antes de serem desencadeadas ações corretivas, Schmidgall et al. (1996) notaram que nos custos de alimentação e de bebidas, o controlo é muito mais apertado nos EUA, ocorrendo o inverso noutras categorias, exceto nos gastos com o pessoal, em que o controlo é semelhante.

Schmidgall e Ninemeier (1987) e Schmidgall et al. (1996) investigaram ainda quais as consequências do incumprimento dos objetivos, sendo o principal a redução ou eliminação de bónus. Mas na Escandinávia, segundo Schmidgall et al. (1996), as consequências não se fazem sentir diretamente a nível pessoal, traduzindo-se antes em ajustamentos operacionais globais.

Na Grécia, Makrigiannakis \& Soteriades (2007) observaram que a maioria dos hotéis monitoriza os custos com um desvio aceitável de cinco por cento; alguns hotéis toleram um maior desvio em alimentação, pessoal e outros custos.

No Reino Unido, Jones (2006) constatou que $71 \%$ das organizações estabelece benchmarks que irão desencadear a investigação dos desvios orçamentais. Aquando da deteção de desvios, a tomada de decisões corretivas cabe aos gestores com responsabilidade direta àquele nível e ao seu gestor de linha, sem prejuízo da análise de desvios também envolver os contabilistas e os controllers.

Em Portugal, na organização estudada por Cruz (2007), a monitorização dos valores reais versus orçamentados é feita mensalmente. Estes valores e os respetivos desvios constam das demonstrações operacionais mensais e, quando significativos, devem ser justificados pelo diretor hoteleiro.

A necessidade de mais investigação sobre as práticas de orçamentação das organizações de serviços, como os hotéis, tem sido enfatizada (Schmidgall et al., 1996; Jones, 1998; Sharma, 2002; Cruz, 2007; Uyar \& Bilgin, 2011; Souza \& Lunkes, 2015). Reconhece-se que os orçamentos são muito utilizados. São essenciais para o planeamento e controlo, e ainda para a avaliação de desempenho, comunicação, coordenação e motivação do pessoal. Está por fazer o levantamento exaustivo das práticas de orçamentação dos hotéis em Portugal; os estudos realizados abordam marginalmente a temática. Este estudo contribui para o preenchimento desta lacuna, apresentando evidência empírica relativa à principal região turística portuguesa - o Algarve.

\section{Metodologia}

\subsection{Questões de investigação}

Este estudo analisa as práticas de orçamentação dos hotéis localizados no Algarve. Note-se que faz parte de um trabalho de âmbito mais vasto que investiga as práticas de contabilidade de gestão dos hotéis do Algarve (Faria, 2012).

Tendo por base a revisão da literatura, formularam-se as seguintes questões de investigação: 
- Q11: Os hotéis elaboram orçamentos?

- Q12: Qual o horizonte temporal dos orçamentos?

- Q13: Por que razões se elaboram orçamentos?

- Q14: Quando se inicia a elaboração do orçamento anual?

- Q15: Qual a abordagem utilizada no processo orçamental?

- QI6: Que tipo de informação serve de base à elaboração do orçamento anual?

- Q17: Os hotéis elaboram orçamentos de base zero?

- Q18: Os hotéis elaboram orçamentos flexíveis? Por que motivos não os elaboram?

- QI9: Os hotéis reveem os orçamentos?

- QI10: Os hotéis procedem à análise de desvios orçamentais? Quais os termos de comparação das realizações?

Para responder a estas questões procedeu-se à análise dos dados através da aplicação de estatística descritiva univariada e bivariada, sendo os resultados apresentados através de tabelas de frequências absolutas e relativas e calculadas características amostrais (e.g., média). Para compreender a relação entre variáveis recorreu-se ainda à análise multivariada (e.g., análise fatorial).

\subsection{Universo e amostra}

O estudo circunscreve-se aos hotéis e aos hotéis-apartamentos (doravante designados por hotéis), de categoria quatro e cinco estrelas, e à região algarvia, pela sua importância e identidade única como destino turístico. Nos últimos anos, o Algarve tem mantido a sua posição como principal destino em Portugal, sendo, em 2017, a região com maior capacidade (camas) de alojamento a nível nacional (36,8\% das camas totais), com maior oferta de estabelecimentos hoteleiros ( $22,2 \%$ do total), com maior número de dormidas (33,8\% das dormidas totais), e a seguir a Lisboa, a região com maior número de hóspedes (INE, I.P., 2018). Popularmente conhecido, o Algarve é um caso particular no contexto do turismo nacional, atentas as suas características. Apresenta elevada sazonalidade (refletida na baixa taxa de ocupação-cama comparativamente a outras regiões turísticas como Madeira e Lisboa), com a procura concentrada nos meses de verão e no produto "Sol e Praia". A acentuada sazonalidade representa um risco económico acrescido em virtude da volatilidade que provoca nas receitas, em confronto com o peso elevado dos custos fixos na estrutura de custos, exigindo uma redobrada atenção e acompanhamento para uma gestão eficiente dos recursos, podendo aqui os orçamentos, como instrumento de controlo, desempenhar um papel essencial.

Para a identificação do universo foi solicitada a base de dados da Associação dos Hotéis e Empreendimentos Turísticos do Algarve (AHETA). A dimensão do universo fixou-se em 89 hotéis ( 57 hotéis e 32 hotéis-apartamentos). Todos os hotéis foram contactados; a taxa de resposta fixou-se em $74,2 \%$.

Dos 66 hotéis inquiridos, $81,8 \%$ possuem categoria quatro estrelas e $18,2 \%$ categoria cinco estrelas. Quase $70 \%$ pertencem à tipologia hotéis; os restantes $30 \%$ são hotéis-apartamentos. Quanto ao tipo de gestão, 68,1\% são geridos por cadeias hoteleiras (nacionais, 54,5\%; internacionais, 13,6\%); 31,9\% são hotéis independentes. O regime de exploração é, na maioria 
dos hotéis, gestão própria (53\%), encontrando-se $27,3 \%$ com contrato de arrendamento, 9,1\% com contrato de exploração e 10,6\% com contrato de gestão.

É de salientar que os principais grupos hoteleiros a operar em Portugal se encontram representados na amostra.

\subsection{Método de pesquisa, recolha de dados e respondentes}

Recolheram-se dados primários através de um questionário estruturado dirigido aos responsáveis pela área contabilística/financeira. A validade do conteúdo foi assegurada pela sua fundamentação na revisão da literatura e pelo recurso a medidas usadas em questionários de referência na área da contabilidade de gestão e da gestão hoteleira (e.g., Chenhall \& Langfield-Smith, 1998; Jones, 1998, 2006; Mia \& Patiar, 2001; Pavlatos \& Paggios, 2009), assim como pela revisão crítica por peritos na área e potenciais respondentes.

O receio de que um inquérito postal ou eletrónico pudesse gerar uma amostra demasiado pequena para produzir uma análise de dados significativa e o facto de a dimensão do universo permitir a deslocação a todos os hotéis conduziu à opção pela recolha presencial. Através de contato telefónico foram identificados os responsáveis pela contabilidade de gestão, tendo-lhes sido apresentados os objetivos da investigação e garantida a confidencialidade dos dados. Após a sua concordância em participar no estudo foram agendadas reuniões para a recolha dos dados. Admite-se que os inconvenientes associados à recolha presencial (e.g., possibilidade de criar enviesamento nas respostas pela possibilidade de o pesquisador induzir a própria resposta do inquirido e elevado consumo de tempo) tenham sido mitigados, pois julga-se ter contribuído para um maior número de questionários preenchidos e para uma redução no número de perguntas sem resposta desvantagens geralmente associadas aos questionários enquanto instrumento de recolha de dados. Também contribuiu para uma maior fiabilidade das respostas, pois permitiu esclarecer os inquiridos sobre o significado de determinados conceitos e ajudou-os na compreensão das questões. Possibilitou ainda a visualização in loco dos sistemas, contribuindo assim para uma melhor perceção dos mesmos. Uma vez que se previa a dificuldade dos inquiridos em compreender a terminologia própria da contabilidade de gestão, o questionário tinha em anexo um glossário.

Foi preenchido um único questionário nos grupos hoteleiros com função financeira centralizada, ou seja, com um diretor financeiro comum a todos os hotéis do grupo e com idênticos procedimentos e práticas contabilísticas. Responderam ao questionário 35 indivíduos, exercendo essencialmente funções de direção na área financeira ou administrativa.

\section{Resultados}

\subsection{Elaboração de orçamentos}

Apenas quatro hotéis $(6,1 \%)$, todos independentes, não elaboram orçamentos. Destes, um elaborou orçamentos no passado; os restantes, pertencentes ao mesmo dono, tencionam elaborá-los no futuro.

Este resultado corrobora os de vários estudos que apontam para uma elevada utilização da técnica no setor (Jones, 1998; 2006; Makrigiannakis \& Soteriades, 2007; Santos et al., 2010). 
Os 62 hotéis elaboram, todos eles, orçamentos operacionais anuais, que se dividem em 12 períodos de um mês. Nenhum hotel tem o orçamento repartido em treze períodos de quatro semanas; porém, um hotel pertencente a uma cadeia internacional indicou que a sede trabalha com 13 períodos.

Mais de $90 \%$ dos hotéis têm as receitas e os gastos desdobrados por departamento. A estrutura do orçamento é, regra geral, idêntica à da conta de exploração, facilitando comparações mensais entre os valores reais e os valores orçamentados, o que está em consonância com Uyar e Bilgin (2011), que também mencionam a elaboração de relatórios mensais durante o período orçamental.

\subsection{Horizonte temporal}

Relativamente ao horizonte temporal, apenas $21 \%$ dos hotéis elaboram orçamentos a mais de um ano. É nas cadeias internacionais que se verifica a maior taxa de elaboração de orçamentos a longo prazo (44,4\%). A taxa de elaboração nos hotéis pertencentes a cadeias nacionais (16,7\%) é ligeiramente inferior à dos independentes (17,6\%). A distribuição cruzada desta variável com a categoria revela que a proporção de hotéis de cinco estrelas (50\%) que elaboram orçamentos a longo prazo é muito superior à de hotéis de quatro estrelas (15,4\%).

Quanto ao horizonte do orçamento a longo prazo, observou-se uma maior frequência no prazo de cinco anos (sete, ou seja, 53,8\% do total de hotéis). Este é o prazo predominante nas cadeias nacionais. Metade dos hotéis pertencentes a cadeias internacionais elaboram igualmente orçamentos a cinco anos, enquanto os restantes elaboram-nos para um período de três anos.

Em todos os hotéis, o orçamento a longo prazo integra o orçamento operacional/de exploração, em $92,3 \%$ o orçamento de investimentos e em $84,6 \%$ o orçamento de tesouraria. Regra geral, o orçamento operacional a longo prazo coincide no primeiro ano com o orçamento anual; seguidamente não é tão detalhado.

A maioria dos hotéis ( $87 \%$ ) não elabora orçamentos contínuos (rolling budgets); sete (11,3\%) mantêm em sistema de orçamentação contínua o orçamento a longo prazo e um (1,6\%) o orçamento de tesouraria. Não existem grandes diferenças entre cadeias e independentes; porém, os independentes registam a maior percentagem de não elaboração e as cadeias internacionais, a menor.

Alguns hotéis elaboram, em conjunto com o orçamento anual, rolling forecasts, que lhes permitem antever, a qualquer momento, os próximos 12 ou mais meses, como um hotel pertencente a uma cadeia internacional que à data da recolha de dados dispunha constantemente de forecasts com o mesmo nível de detalhe do orçamento para os 18 meses seguintes. Porém, num contato posterior, a mesma unidade informou já só dispor de forecasts para 12 meses.

É de supor que o contexto de incerteza e permanente volatilidade então vivenciados tenham contribuído para o abandono de técnicas de orçamentação a longo prazo.

Observou-se que a quase totalidade $(98,4 \%)$ dos hotéis possui o mesmo suporte informático para a contabilidade financeira e para a contabilidade de gestão. Cerca de $90 \%$ utiliza folhas de cálculo, designadamente para a elaboração dos orçamentos. Note-se que também Uyar e Bilgin (2011) e Souza e Lunkes (2015) reportaram um elevado uso do Microsoft Excel para fins orçamentais. 


\subsection{Razões para a elaboração de orçamentos}

O Quadro 2 revela que os orçamentos são muito utilizados para avaliar o desempenho, definir objetivos e metas, auxiliar o controlo, auxiliar o planeamento a curto prazo e motivar a gestão e o pessoal. Mais de $60 \%$ dos hotéis faz pouco uso dos orçamentos para auxiliar o planeamento a longo prazo, o que é consistente com a reduzida taxa de elaboração de orçamentos a mais de um ano.

Quadro 2. Razões para a elaboração dos orçamentos

\begin{tabular}{|c|c|c|c|c|c|c|c|}
\hline Finalidades & $\mathrm{N}$ & $\begin{array}{c}\text { Nenhum } \\
\text { uso } \\
1\end{array}$ & $\begin{array}{c}\text { Reduzido } \\
\text { uso } \\
2\end{array}$ & $\begin{array}{c}\text { Algum } \\
\text { uso } \\
3\end{array}$ & $\begin{array}{c}\text { Uso } \\
\text { elevado } \\
4\end{array}$ & $\begin{array}{l}\text { Uso muito } \\
\text { elevado } \\
5\end{array}$ & Média \\
\hline Planeamento a curto prazo & 62 & - & $1,6 \%$ & $16,1 \%$ & $41,9 \%$ & $40,3 \%$ & 4,21 \\
\hline Planeamento a longo prazo & 62 & $12,9 \%$ & $24,2 \%$ & $25,8 \%$ & $11,3 \%$ & $25,8 \%$ & 3,13 \\
\hline Definir objetivos e metas & 62 & - & $3,2 \%$ & $8,1 \%$ & $38,7 \%$ & $50,0 \%$ & 4,35 \\
\hline Motivar a gestão e o pessoal & 62 & $1,6 \%$ & $8,1 \%$ & $11,3 \%$ & $54,8 \%$ & $24,2 \%$ & 3,92 \\
\hline Comunicar planos & 62 & $3,2 \%$ & $8,1 \%$ & $40,3 \%$ & $32,3 \%$ & $16,1 \%$ & 3,50 \\
\hline Coordenar as atividades & 62 & $1,6 \%$ & $8,1 \%$ & $35,5 \%$ & $40,3 \%$ & $14,5 \%$ & 3,58 \\
\hline Auxiliar o controlo & 62 & - & $1,6 \%$ & $11,3 \%$ & $51,6 \%$ & $35,5 \%$ & 4,21 \\
\hline Avaliar o desempenho & 62 & $1,6 \%$ & $4,8 \%$ & - & $43,5 \%$ & $50,0 \%$ & 4,35 \\
\hline
\end{tabular}

Comparando os resultados obtidos com os de Jones (1998, 2006), no Reino Unido e com os de Uyar e Bilgin (2011), na Turquia, não se observam grandes diferenças. Avaliar o desempenho, auxiliar o controlo e motivar a gestão e o pessoal estão entre os principais motivos para a produção de orçamentos. No entanto, neste estudo e no de Uyar e Bilgin (2011), os orçamentos são igualmente encarados como um importante auxiliar do planeamento a curto prazo, o que não sucede nas organizações hoteleiras do Reino Unido (Jones, 1998, 2006), apesar de "auxiliar o planeamento" ser a razão para os orçamentos mais citada nos livros de texto, como nota Jones (2006). A autora refere que a justificação poderá residir no facto do planeamento ocorrer muito antes do momento em que os orçamentos são elaborados. No presente estudo os hotéis também utilizam menos os orçamentos como ferramenta de planeamento a longo prazo do que os do Reino Unido (Jones, 1998, 2006) e da Turquia (Uyar \& Bilgin, 2011).

\subsection{Antecedência com que se inicia o orçamento anual}

Mais de $80 \%$ dos hotéis começam a elaborar o orçamento um a três meses antes. Os que iniciam o processo mais cedo pertencem a cadeias internacionais (um terço no terceiro trimestre do exercício anterior e 11,1\% ainda no primeiro semestre desse mesmo período), o que se justifica pelo tempo que é necessário até que seja aprovado pelos serviços centrais. Apesar de 5,9\% dos hotéis independentes iniciarem o processo até um mês antes, não se observam grandes diferenças entre cadeias nacionais e independentes. De realçar que nenhum hotel inicia o processo no próprio exercício. 
Os resultados corroboram os de Planas (2004), em Espanha, que também constatou que as cadeias internacionais iniciam o processo com maior antecedência.

\subsection{Abordagem utilizada no processo orçamental}

Verifica-se que a abordagem mais utilizada é a top-down ou descendente ( 32 hotéis, 51,6\%), seguida pela abordagem bottom-up ou ascendente ( 16 hotéis, $25,8 \%$ ) e pela combinação das duas abordagens (14 hotéis, 22,6\%) (Gráfico 1).

Gráfico 1. Abordagem utilizada por tipo de gestão

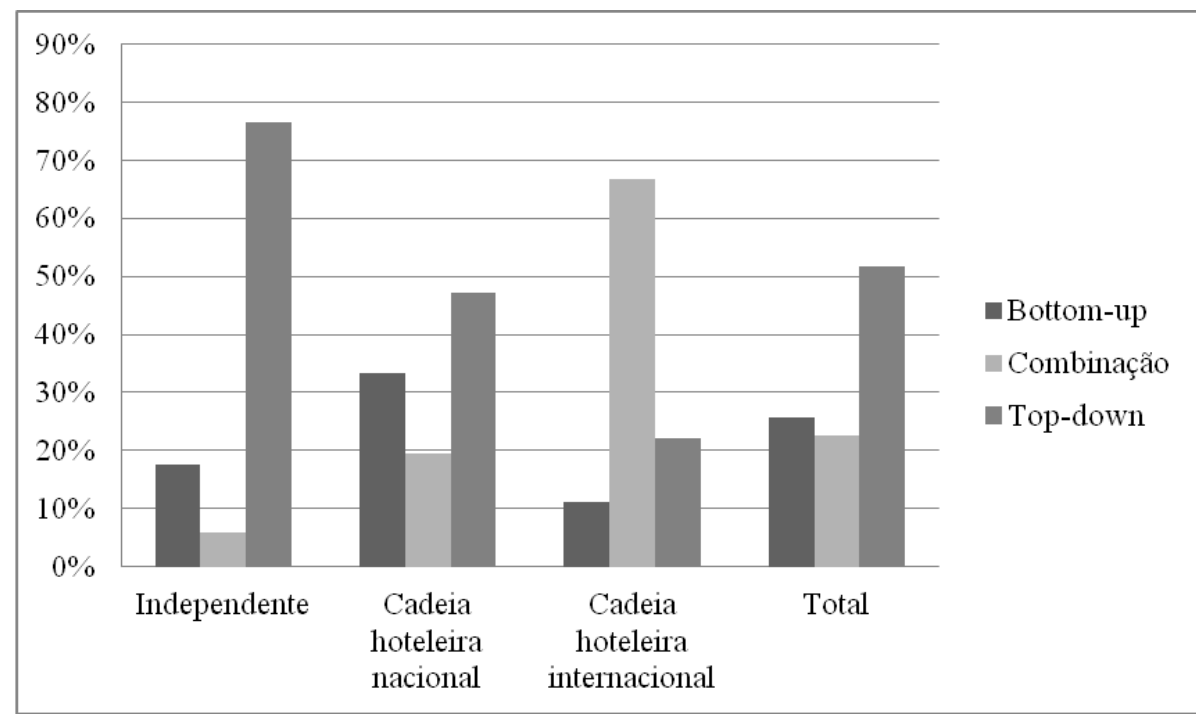

Considerando o tipo de gestão, nos hotéis pertencentes a cadeias internacionais predomina a combinação das duas abordagens $(66,7 \%)$. Tal significa que a sede estabelece metas ou diretrizes (tendo por base o Gross Operating Profit - GOP) mas os orçamentos são produzidos numa perspetiva bottom-up, ou seja, com a participação dos gestores a níveis mais baixos. Nos hotéis pertencentes a cadeias nacionais e nos independentes impera a abordagem top-down ( $47,2 \%$ e $76,5 \%$, respetivamente).

Em alguns hotéis pertencentes a cadeias foi referida a existência de comités (e.g., senior committee, executive committee) em que participam o diretor geral, o diretor financeiro, o diretor de vendas/marketing, os diretores de departamento, o diretor de revenue management e o diretor de recursos humanos, entre outros. De notar que também Uyar e Bilgin (2011) e Souza e Lunkes (2015) fazem referência à existência de budget committees em $87,3 \%$ e $45 \%$, respetivamente dos hotéis inquiridos.

Relativamente ao grau de envolvimento dos diferentes níveis hierárquicos na elaboração do orçamento, constata-se que os diretores gerais e os diretores financeiros têm um envolvimento maior (médias de 4,6 e 4,13, respetivamente, numa escala de 1-Reduzido a 5-Elevado). A participação dos diretores de departamento ou dos chefes de secção é reduzida, mas coerente com a constatação de que a abordagem mais utilizada pela generalidade dos hotéis é a top-down. O envolvimento dos donos no processo é ainda menor: limitam-se, na maioria dos casos, a aprovar o orçamento. 
Para tentar perceber o tipo de correlações existente entre as variáveis, procedeu-se a uma Análise Fatorial pelo método das componentes principais onde se juntaram as variáveis associadas à dimensão com as variáveis que mostram o envolvimento das diversas chefias (Quadro 3).

Quadro 3. Análise fatorial das variáveis "dimensão" e "envolvimento dos diretores"

\begin{tabular}{lccc}
\hline & \multicolumn{3}{c}{ Fatores } \\
& 1 & 2 & 3 \\
\hline Fator dimensão & 0,985 & & \\
Número de quartos & 0,948 & & \\
Número de camas & 0,934 & & \\
Número de trabalhadores (média 2008) & 0,797 & 0,290 & \\
Envolvimento do diretor financeiro & & 0,855 & \\
Envolvimento dos diretores de departamento & & 0,747 & \\
Envolvimento do diretor geral & & 0,636 & $-0,587$ \\
Envolvimento dos donos & & 0,890 \\
\hline
\end{tabular}

O quadro ilustra a relação entre as oito variáveis e os três principais fatores extraídos pelo método dos componentes principais com rotação "Varimax". Existem três fatores preponderantes que são capazes de explicar uma boa proporção $(80,2 \%)$ da variabilidade da amostra. $O$ primeiro fator exprime a dimensão pura; o segundo fator mostra o envolvimento dos diretores; o terceiro fator mostra o envolvimento dos donos. Constata-se que o número de trabalhadores ( $1 .^{\circ}$ fator) também se encontra bem representado no $2 .^{\circ}$ fator (factor loading $=0,29)$ e o envolvimento do diretor geral encontra-se representado também no $3 .^{\circ}$ fator, mas com um factor loading de sinal oposto $(-0,59)$. Assim, quanto mais o dono se envolve, menos o fará o diretor geral e vice-versa.

\subsection{Informação utilizada na elaboração do orçamento anual}

Conforme se observa no Quadro 4, os orçamentos baseiam-se sobretudo nos valores reais do ano anterior, mas também em análises de mercado internas (em alguns casos, feitas de forma rudimentar) e em indicadores económicos locais/nacionais/internacionais. Em cerca de $55 \%$ dos hotéis, os valores orçamentados do ano anterior têm reduzido ou nenhum uso. Constatou-se que alguns hotéis também recorrem aos forecasts ou reforecasts (e.g., valores acumulados à data mais os forecasts até ao final do ano). Os resultados corroboram os de Jones (1998, 2006), Ivankovic e Jerman (2011) e Uyar e Bilgin (2011), embora os hotéis turcos também utilizem os valores orçamentados do ano anterior. 
Quadro 4. Informação utilizada na elaboração do orçamento anual

\begin{tabular}{|c|c|c|c|c|c|c|c|}
\hline & $\mathrm{N}$ & $\begin{array}{c}\text { Nenhum } \\
\text { uso } \\
1\end{array}$ & $\begin{array}{c}\text { Reduzido } \\
\text { uso } \\
2\end{array}$ & $\begin{array}{c}\text { Algum } \\
\text { uso } \\
3\end{array}$ & $\begin{array}{c}\text { Uso } \\
\text { elevado } \\
4\end{array}$ & $\begin{array}{c}\text { Uso } \\
\text { muito } \\
\text { elevado } \\
5\end{array}$ & Média \\
\hline Valores reais do ano anterior & 62 & - & - & - & $38,7 \%$ & $61,3 \%$ & 4,61 \\
\hline $\begin{array}{l}\text { Valores orçamentados do ano } \\
\text { anterior }\end{array}$ & 62 & $29,0 \%$ & $25,8 \%$ & $24,2 \%$ & $19,4 \%$ & $1,6 \%$ & 2,39 \\
\hline Análises de mercado internas & 62 & $3,2 \%$ & $16,1 \%$ & $6,5 \%$ & $54,8 \%$ & $19,4 \%$ & 3,71 \\
\hline $\begin{array}{l}\text { Indicadores económicos } \\
\text { locais/nacionais/internacionais }\end{array}$ & 62 & $1,6 \%$ & $22,6 \%$ & $19,4 \%$ & $27,4 \%$ & $29,0 \%$ & 3,60 \\
\hline Estatísticas da indústria & 62 & $11,3 \%$ & $41,9 \%$ & $14,5 \%$ & $21,0 \%$ & $11,3 \%$ & 2,79 \\
\hline
\end{tabular}

As estatísticas da indústria não são praticamente utilizadas. Os que referiram dar-lhes uso elevado mencionaram as de associações hoteleiras, disponíveis gratuitamente, e as produzidas por empresas de consultoria internacional. Poucos hotéis pagam este tipo de estudos, não dispondo assim de informação sobre os concorrentes. Em regra, pertencem a cadeias internacionais.

\subsection{Elaboração de orçamentos de base zero}

Verifica-se que $74,2 \%$ dos hotéis nunca elaboraram orçamentos de base zero, 8,1\% fazem-nos apenas quando não existe histórico (e.g., novos hotéis, projetos, ... ) e 6,5\% quando a situação o exige (e.g., redução nas taxas de ocupação, crise, ...). Somente $4,8 \%$ dos hotéis recorrem frequentemente à orçamentação de base zero. Os hotéis pertencentes a cadeias hoteleiras internacionais são os que mais utilizam esta técnica e os independentes os que a ela menos recorrem.

Os resultados relativos à utilização de orçamentos de base zero estão em consonância com os reportados noutros estudos, que revelam que esta técnica não é muito utilizada no setor (Schmidgall et al., 1996; Jones, 1998, 2006; Planas, 2004; Cruz, 2007; Uyar \& Bilgin, 2011; Souza \& Lunkes, 2015).

\subsection{Elaboração de orçamentos flexíveis}

$\mathrm{Na}$ indústria hoteleira, a envolvente é incerta e em tais circunstâncias os orçamentos flexíveis são uma das mais importantes ferramentas de controlo disponíveis. Porém, os hotéis utilizam-nos pouco: $90,3 \%$ dos hotéis que elaboram orçamentos não consideram vários níveis de atividade. As cadeias hoteleiras internacionais são as que mais utilizam esta ferramenta $(44,4 \%)$, seguindo-se os independentes (11,8\%). O respondente de uma cadeia internacional comentou: "a maior parte dos custos varia com a ocupação. Temos 36 ficheiros para um orçamento".

Quanto aos motivos para a não elaboração de orçamentos flexíveis, $25 \%$ dos hotéis indicaram o elevado peso dos custos fixos nos custos totais e 10,7\% (apenas hotéis independentes e cadeias nacionais) apontaram os elevados custos comparativamente aos benefícios. Mais de metade dos hotéis $(53,6 \%)$ apresentaram outras razões para não elaborarem orçamentos flexíveis, como sejam "não exigência por parte da 
empresa/administração"; "falta de oportunidade"; "assunto não abordado/equacionado"; "desconhecimento da técnica"; "desvio do objetivo a atingir"; "não é visto como ferramenta prioritária"; dificuldade em separar custos fixos e variáveis"; "facilidade de mudar os pressupostos"; "o sistema não está preparado"; "a dimensão não justifica"; "utilizamos os forecasts para reagir à mudança".

Os resultados corroboram os de outros estudos que concluem que os orçamentos flexíveis não são muito utilizados no setor hoteleiro (Collier \& Gregory, 1995; Jones, 1998, 2006; Pavlatos \& Paggios, 2009; Souza \& Lunkes, 2015).

\subsection{Revisão do orçamento}

Quase 60\% dos hotéis que elaboram orçamentos procedem à sua revisão, sendo a proporção de hotéis geridos por cadeias internacionais que a fazem mais elevada $(88,9 \%)$ do que a de cadeias nacionais $(61,1 \%)$ e independentes ( $41,2 \%)$.

Constatou-se, porém, que em alguns hotéis que assinalaram rever o orçamento, o que ocorre é uma reavaliação/atualização (mensal ou trimestral) dos números através de forecasts ou reforecasts; o orçamento original não é alterado.

Assim, alguns dos respondentes que apontaram o orçamento como suscetível de revisão, estavam a referir-se à prática de "reforecasting", ou seja, "à revisão da informação operacional real (normalmente da demonstração dos resultados) para avaliar se são necessárias modificações no orçamento original” (Schmidgall \& Ninemeier, 1987: 82). Estas alterações são traduzidas em forecasts ou reforecasts, que partem dos valores do orçamento e, em regra, possuem o mesmo nível de pormenor. Em certos hotéis, dada a sua importância integram, juntamente com os valores reais e os valores orçamentados originalmente, os relatórios/demonstrações de resultados mensais.

Os resultados obtidos estão em linha com os de Schmidgall e Ninemeier (1987) para as cadeias hoteleiras dos EUA (70\%) e por Makrigiannakis e Soteriades (2007) para a Grécia, onde a maioria dos hotéis revê o orçamento. Estes resultados são superiores aos reportados por Schmidgall et al. (1996) para a Escandinávia e Schmidgall e DeFranco (1998) para os EUA, onde se conclui que apenas $44 \%$ dos hotéis e um em cada quatro respondentes, respetivamente, revê o orçamento. Estão também em consonância com as conclusões de Cruz (2007:585) que refere que, face à cada vez maior competitividade nos mercados, as práticas de orçamentação e controlo orçamental estão a ser reestruturadas de modo a serem usadas de uma forma mais flexível, o que se traduz na revisão mais frequente do orçamento anual e no uso de rolling forecasts a nível do hotel.

No que toca à periodicidade da revisão do orçamento (mensal, trimestral, anual, quando necessário), não se observam diferenças entre os hotéis, conclusão a que também chegaram Souza e Lunkes (2015). Atendendo ao tipo de gestão, verifica-se que a maioria (87,5\%) dos hotéis pertencentes a cadeias internacionais revê o orçamento mensalmente, as cadeias nacionais fazem-no mais espaçadamente: $40,9 \%$ fazem-no uma vez por ano e em $27,3 \%$ destas unidades a revisão do orçamento ocorre somente quando o desempenho mostra ser necessário (por exemplo, "quando há grandes derrapagens" ou "quando os desvios são significativos"). De realçar que cerca de $43 \%$ dos independentes também o fazem apenas nessas circunstâncias; outros tantos reveem o orçamento trimestralmente. 


\subsection{Controlo orçamental}

Observou-se que a análise de desvios tem um uso aceitável (média de 3,57, numa escala de 1Nenhum uso a 5-Uso muito elevado), o que corrobora Gomes (2007) e Santos et al. (2010), que identificam os "orçamentos" e a "análise de desvios" como as técnicas tradicionais mais utilizadas em Portugal.

Quantos aos termos de comparação das realizações, os hotéis utilizam sobretudo os valores reais do ano anterior (média de 4,74 ) e os valores constantes do orçamento original (média de 4,6). Cerca de 95\% dos hotéis comparam "frequentemente" ou "sempre" os valores reais com os valores orçamentados inicialmente. Sendo "avaliar o desempenho" um dos principais motivos que levam os hotéis participantes no estudo a elaborar orçamentos, não surpreende que a grande maioria (83,3\%) atribua a este indicador uma importância elevada ou muito elevada para efeitos de avaliação do desempenho e que, em cerca de $80 \%$ dos hotéis, os sistemas de recompensa se baseiem principalmente em indicadores financeiros, designadamente na comparação dos valores reais com os valores orçamentados, o que está em consonância com a literatura analisada (Schmidgall \& Ninemeier, 1987; Collier \& Gregory, 1995; Cruz, 2007).

Embora menos, os valores do orçamento revisto/forecasts são também utilizados para efeitos de comparação (média de 3,73). Estes resultados corroboram os de Schmidgall e Ninemeier (1987) e de Schmidgall et al. (1996).

Quase dois terços dos hotéis nunca ou raramente compara os valores reais com custos padrões (média de 2,26). Estes são utilizados "sobretudo no departamento de A\&B", "para a rendibilidade departamental" e "mais a nível global”. Aliás, um pouco mais de dois terços dos hotéis $(68,2 \%)$ inquiridos "nunca" ou "raramente" usa o custeio padrão, o que vai ao encontro dos resultados de Ivankovic e Jerman (2011).

Os valores reais também são pouco comparados com os valores da concorrência: metade dos hotéis "nunca" ou "raramente" os utiliza (média de 2,52). Os que o fazem, comparam sobretudo receitas e preços médios (Average Daily Rate - ADR; Revenue per Available Room - REVPAR), através de sítios de reservas ou de comparação de preços na internet. Também Cruz (2007) constatou que o benchmarking externo realizado na cadeia internacional analisada assentava somente em variáveis relacionadas com a receita (taxa de ocupação, ADR e REVPAR), não incluindo uma análise sistemática das demonstrações financeiras e dos custos da concorrência.

Conclui-se, deste modo, que os hotéis não possuem informação que lhes permita avaliar a sua posição relativamente aos concorrentes e, consequentemente, formular estratégias.

\section{Conclusões}

Este estudo contribui para o conhecimento e compreensão das práticas de orçamentação dos hotéis em Portugal, ao proporcionar evidência sobre a região do Algarve. Região que, nos últimos anos, tem sido o principal destino turístico português e que apresenta uma característica peculiar que a distingue das demais: uma elevada sazonalidade que provoca flutuação nas taxas de ocupação ao longo do ano.

Embora os orçamentos não estejam isentos de críticas, são a técnica de contabilidade de gestão mais utilizada na indústria hoteleira (Collier \& Gregory, 1995; Schmidgall et al., 1996; Jones, 1998, 2006; Pavlatos \& Paggios, 2009; Santos et al., 2010). 
Os resultados confirmam que apenas quatro $(6,1 \%)$ dos 66 hotéis de quatro e cinco estrelas do Algarve não elaboram orçamentos. Os que elaboram orçamentos operacionais anuais subdividem-nos em doze períodos mensais. Apenas $21 \%$ dos hotéis elaboram orçamentos a mais de um ano; são essencialmente hotéis pertencentes a cadeias internacionais e de categoria cinco estrelas. Embora poucos hotéis elaborem rolling budgets, é frequente a produção de rolling forecasts em hotéis pertencentes a cadeias hoteleiras.

Os orçamentos servem principalmente para avaliar o desempenho, definir objetivos e metas, auxiliar o controlo e o planeamento a curto prazo. São pouco utilizados no planeamento a longo prazo, o que está em consonância com a reduzida taxa de elaboração de orçamentos a mais de um ano.

A abordagem mais utilizada na elaboração dos orçamentos é a top-down ou descendente, sendo a incidência desta abordagem nos hotéis independentes elevada. Nas cadeias internacionais predomina a combinação das duas abordagens. Os diretores gerais e os diretores financeiros são os níveis hierárquicos com maior envolvimento no processo orçamental. A participação dos diretores de departamento/chefes de secção é reduzida.

Mais de $80 \%$ dos hotéis começam a elaborar os orçamentos com antecedência de um a três meses. As cadeias internacionais iniciam-nos mais cedo.

Os orçamentos (anuais) têm por base essencialmente os valores reais do ano anterior, análises de mercado feitas internamente e indicadores económicos. Os valores orçamentados do ano anterior têm pouco uso.

A orçamentação de base zero e os orçamentos flexíveis também são pouco utilizados. Os hotéis pertencentes a cadeias internacionais são os que mais recorrem a estas ferramentas.

Quase 60\% dos hotéis reveem o orçamento, sendo a proporção de hotéis pertencentes a cadeias internacionais que o fazem mais elevada. Como termo de comparação com os valores reais para efeitos de análise de desvios, os hotéis utilizam essencialmente os valores reais do ano anterior e os valores do orçamento original. A comparação dos valores orçamentados com os valores reais é muito utilizada na avaliação do desempenho e está na base dos sistemas de recompensa.

Os resultados estão em linha com os reportados na literatura de contabilidade de gestão confirmando que os orçamentos são uma das técnicas mais utilizadas e uma das mais valiosas ferramentas de planeamento e controlo e de avaliação do desempenho na indústria hoteleira.

O estudo contribui para o conhecimento e para a compreensão das práticas de orçamentação em uso nos hotéis de quatro e de cinco estrelas localizados no Algarve (Portugal) e amplia a literatura sobre orçamentação no setor hoteleiro, ao evidenciar o elevado uso de orçamentos, a sua utilização com fins de avaliação de desempenho e controlo e o reduzido uso de orçamentos flexíveis e de orçamentos de base zero, uma conclusão transversal a diversos estudos, independentemente do país de origem. Por fim, identifica diferenças entre as práticas de orçamentação dos hotéis pertencentes a cadeias internacionais e os restantes hotéis no tocante à utilização de orçamentos a longo prazo, à antecedência com que iniciam a elaboração dos orçamentos, à abordagem utilizada, à periodicidade com que reveem os orçamentos e à elaboração de orçamentos contínuos, de orçamentos flexíveis e de orçamentos de base zero, o que poderá indiciar que nas cadeias internacionais o processo de orçamentação é mais sofisticado. 
Como principal limitação do estudo aponta-se o facto de este se circunscrever apenas à região algarvia, pelo que se sugere, para investigação futura, alargar o estudo a outro universo, nomeadamente a Portugal continental e insular, e a regiões com sazonalidade menos acentuada.

Em estudo posterior pretende-se identificar os fatores que influenciam as práticas de orçamentação na indústria hoteleira, tais como a dimensão, a afiliação a cadeia hoteleira e a intensidade da concorrência. Os aspetos comportamentais associados ao processo orçamental são outra área a merecer aprofundamento.

\section{Referências}

Brown, J. B. (1995). Management control in the hospitality industry: Behavioural implications. In P. Harris (Ed.), Accounting and Finance for the International Hospitality Industry (pp. 183-201). Oxford: Butterworth-Heinemann.

Bunce, P., Fraser, R. \& Woodcock, L. (1995). Advanced budgeting: A journey to advanced management systems. Management Accounting Research, 6(3), 253-265. doi:10.1006/mare.1995.1017

Chenhall, R. \& Langfield-Smith, K. (1998). Adoption and benefits of management accounting practices: An Australian study. Management Accounting Research, 9(1), 1-19. doi:10.1006/mare.1997.0060

Collier, P. \& Gregory, A. (1995). The practice of management accounting in hotel groups. In P. Harris (ed.), Accounting and Finance for the International Hospitality Industry (pp. 137-159). Oxford: Butterworth-Heinemann.

Cruz, I. (2007). How might hospitality organizations optimize their performance measurement systems? International Journal of Contemporary Hospitality Management, 19(7), 574-588. doi:10.1108/09596110710818310.

Dugdale, D. (1994). Theory and practice: The views of CIMA members and students. Management Accounting, 72(8), 56-58.

Faria, A. R. (2012). Sistemas de contabilidade de gestão no setor hoteleiro do Algarve: Planeamento ou improvisação? (Tese de doutoramento não publicada). Universidade do Algarve, Faro, Portugal.

Ferreira. A. (2002) Management accounting and control systems design and use: An exploratory study in Portugal (Tese de doutoramento não publicada). The Management School, Lancaster University, Reino Unido.

Gomes, C. (2007). A Contabilidade de gestão e o custeio baseado nas atividades nas grandes empresas portuguesas: Os determinantes do custeio baseado nas atividades (Tese de doutoramento não publicada). Universidade do Minho, Braga, Portugal.

Harris, P. \& Brown, J. B. (1998). Research and development in hospitality accounting and financial management. International Journal of Hospitality Management, 17(2), 161-181. doi:10.1016/S0278-4319(98)00013-9

Hope, J. \& Fraser, R. (2003). Who needs budgets? Harvard Business Review, February 81(2), 108-115.

Instituto Nacional de Estatística (INE), I. P. (2018). Estatísticas do turismo 2017. Disponível em http://www.ine.pt.

Ivankovic, G. \& Jerman, M. (2011). Comparative analysis of budgeting in the Slovene hotel industry. Tourism \& Hospitality Management, 17(1), 91-100.

Jones, T. (1998). UK hotel operators use of budgetary procedures. International Journal of Contemporary Hospitality Management, 10(3), 96-100. doi: 10.1108/09596119810210279

Jones, T. (2006). Budgetary practice within hospitality. In P. Harris \& M. Mongiello (Eds.), Accounting and Financial Management (pp. 72-86). Oxford: Butterworth-Heinemann.

Joshi, P. L. (2001). The international diffusion of new management accounting practices: The case of India. Journal of International Accounting, Auditing and Taxation, 10(1), 85-109. doi:10.1016/S1061-9518(01)00037-4

Joshua, O. \& Mohammed, N. (2013). Budget target setting and effective performance measurement in Nigerian hospitality industry. Journal of Finance \& Economics, 1(3), 39-50. doi:10.12735/jfe.v1i3p39 
Libby, T. \& Lindsay, R. M. (2010). Beyond budgeting or budgeting reconsidered? A survey of NorthAmerican budgeting practice. Management Accounting Research, 21, 56-75. doi:10.1016/j.mar.2009.10.003

Makrigiannakis, G. \& Soteriades, M. (2007). Management accounting in the hotel business: The case of the Greek hotel industry. International Journal of Hospitality and Tourism Administration, 8(4), 47-76. doi:10.1300/J149v08no4_03

Mia, L. \& Patiar, A. (2001). The use of management accounting systems in hotels: An exploratory study. International Journal of Hospitality Management, 20(2), 111-128. doi:10.1016/S02784319(00)00033-5

Pavlatos, O. \& Paggios, I. (2009). Management accounting practices in the Greek hospitality industry. Managerial Auditing Journal, 24(1), 81-98. doi:10.1108/02686900910919910

Planas, F. (2004). La contabilidad de géstion en la industria hotelera: Estudio sobre su Implantación en las Cadenas Hoteleiras en España (Tese de doutoramento não publicada). Universitat Rovira i Virgili, Tarragona, Espanha.

Santos, L., Gomes, C. \& Arroteia, N. (2010). Management accounting practices in the Portuguese lodging industry. European Journal of Tourism, Hospitality and Recreation, 1(1), 110-125.

Schmidgall, R. \& DeFranco, A. (1998). Budgeting and forecasting, current practice in the lodging industry. Cornell Hotel and Restaurant Administration Quarterly, 39(6), 45-51. doi:10.1177/001088049803900609

Schmidgall, R. \& Ninemeier, J. (1987). Budgeting in hotel chains: Co-ordination and control. The Cornell Hotel and Restaurant Administration Quarterly, 28(1), 79-84. doi:10.1177/001088048702800118

Schmidgall, R., Borchgrevink, C. \& Zahl-Begnum, O. (1996). Operations budgeting practices of lodging firms in the United States and Scandinavia. International Journal of Hospitality Management, 15(2), 189-203. doi:10.1016/0278-4319(96)00008-4

Sharma, D. S. (2002). The differential effect of environmental dimensionality, size, and structure on budget system characteristics in hotels. Management Accounting Research, 13, 101-130. doi:10.1006/mare.2002.0183

Souza, P. \& Lunkes, R. J. (2015). Budgeting practices: A study on Brazilian hotel companies. Revista Brasileira de Pesquisa em Turismo, 9(3), 380-399. doi:10.7784/rbtur.v9i3.851

Uyar, A. \& Bilgin, N. (2011). Budgeting practices in the Turkish hospitality industry: An exploratory survey in the Antalya region. International Journal of Hospitality Management, 30(2), 398-408. doi: 10.1016/j.jjhm.2010.07.011

ANA RITA FARIA é doutora em Ciências Económicas e Empresariais (Universidade do Algarve, Portugal) e mestre em Ciências Económicas e Empresariais (Universidade do Algarve). Professora adjunta na Escola Superior de Gestão, Hotelaria e Turismo (Universidade do Algarve) nas áreas da Contabilidade Financeira e da Contabilidade de Gestão Aplicada ao Setor Hoteleiro. Conselheira do Centro de Estudos de História da Contabilidade da Associação Portuguesa de Técnicos de Contabilidade (APOTEC). Investigadora Integrada do Centro de Investigação, Desenvolvimento e Inovação em Turismo - CITUR. Coautora dos livros Contabilidade de gestão hoteleira (ATF Edições, 2016) e Contabilidade gerencial hoteleira (Juruá Editora, 2018) e de capítulos em obras coletivas. Autora de artigos em revistas nacionais e internacionais na área da Contabilidade de Gestão hoteleira e da História da Contabilidade. Endereço institucional: Escola Superior de Gestão, Hotelaria e Turismo, da Universidade do Algarve, Campus da Penha, 8005-139 Faro, Portugal.

LEONOR FERREIRA é professora na Nova School of Business and Economics e na Universidade do Algarve. Doutorada em Gestão de Empresas, mestre em Gestão pelo Instituto Superior de Economia e Gestão (UTL) e licenciada em Administração e Gestão de Empresas pela Universidade Católica Portuguesa. Autora dos livros Financial reporting in Portugal (Routledge, 1994) e Modelos de avaliação de empresas e utilidade da informação contabilística (Ed. Lusíada, 2008) e de capítulos em obras coletivas, tais como La Contabilidad de gestión en Latinoamerica (AECA, 1996), Transnational accounting (Palgrave, 2001) e The Millers' European accounting guide (Aspen, 2003). Orientadora de dissertações de mestrado e doutoramento. Árbitro do CAAD - Centro de Arbitragem Administrativa, membro do Júri de Exame de Admissão à Ordem dos Revisores Oficiais de Contas e membro do Conselho Geral da 
Comissão de Normalização Contabilística. Endereço institucional: Nova School of Business and Economics, Rua Holanda, n. ${ }^{\circ}$ 1, 2775-405, Carcavelos, Portugal.

DUARTE TRIGUEIROS é engenheiro de Telecomunicações e Eletrónica (1970, IST, Lisboa) e trabalhou durante sete anos (1970-1977) no Laboratório de Física e Engenharia Nucleares, depois em empresas industriais como Analista de Sistemas (1977-1986) e finalmente seguiu uma carreira académica (19862013). Doutorou-se na Universidade de East Anglia (1988-1991) e os seus interesses em investigação são Análise de Demonstrações Financeiras e tomada de decisão financeira baseada em Inteligência Artificial. Orientou dissertações de mestrado e doutoramentos na Universidade de Macau, no Instituto Universitário de Lisboa e na Universidade do Algarve. É professor visitante na Universidade de São José em Macau e também investigador do Instituto Universitário de Lisboa. Endereço institucional: ISTAR-IUL, University Institute of Lisbon, Av. Forças Armadas n. ${ }^{\circ}$ 376, 1600-077, Lisboa, Portugal.

Submetido em 7 novembro 2018

Aceite em 11 março 2019 